\title{
Empiric use of anticoagulation in hospitalized patients with COVID-19: a propensity score-matched study of risks and benefits
}

Bo Yu ${ }^{1}$, Victor Perez Gutierrez ${ }^{1}$, Alex Carlos ${ }^{1}$, Gregory Hoge ${ }^{1}$, Anjana Pillai ${ }^{1}$, J. Daniel Kelly ${ }^{2,3,4,5}$ and Vidya Menon ${ }^{1 *}$

\begin{abstract}
Background: Hospitalized patients with COVID-19 demonstrate a higher risk of developing thromboembolism. Anticoagulation (AC) has been proposed for high-risk patients, even without confirmed thromboembolism. However, benefits and risks of AC are not well assessed due to insufficient clinical data. We performed a retrospective analysis of outcomes from AC in a large population of COVID-19 patients.
\end{abstract}

Methods: We retrospectively reviewed 1189 patients hospitalized for COVID-19 between March 5 and May 15, 2020, with primary outcomes of mortality, invasive mechanical ventilation, and major bleeding. Patients who received therapeutic AC for known indications were excluded. Propensity score matching of baseline characteristics and admission parameters was performed to minimize bias between cohorts.

Results: The analysis cohort included 973 patients. Forty-four patients who received therapeutic AC for confirmed thromboembolic events and atrial fibrillation were excluded. After propensity score matching, 133 patients received empiric therapeutic AC while 215 received low dose prophylactic AC. Overall, there was no difference in the rate of invasive mechanical ventilation $(73.7 \%$ versus $65.6 \%, p=0.133)$ or mortality $(60.2 \%$ versus $60.9 \%, p=0.885)$. However, among patients requiring invasive mechanical ventilation, empiric therapeutic AC was an independent predictor of lower mortality (hazard ratio [HR] 0.476, 95\% confidence interval [Cl] 0.345-0.657, $p<0.001$ ) with longer median survival ( 14 days vs 8 days, $p<0.001$ ), but these associations were not observed in the overall cohort $(p=0.063)$. Additionally, no significant difference in mortality was found between patients receiving empiric therapeutic AC versus prophylactic $A C$ in various subgroups with different D-dimer level cutoffs. Patients who received therapeutic AC showed a higher incidence of major bleeding (13.8\% vs 3.9\%, $p<0.001)$. Furthermore, patients with a HAS-BLED score of $\geq 2$ had a higher risk of mortality ( $H R$ 1.482, 95\% Cl 1.110-1.980, $p=0.008$ ), while those with a score of $\geq 3$ had a higher risk of major bleeding (Odds ratio: 1.883, Cl: 1.114-3.729, $p=0.016$ ).

Conclusion: Empiric use of therapeutic AC conferred survival benefit to patients requiring invasive mechanical ventilation, but did not show benefit in non-critically ill patients hospitalized for COVID-19. Careful bleeding risk estimation should be pursued before considering escalation of AC intensity.

\footnotetext{
* Correspondence: menonv@nychhc.org

'Department of Medicine, New York City Health + Hospitals, Lincoln Medical Center, Bronx, New York, USA

Full list of author information is available at the end of the article
}

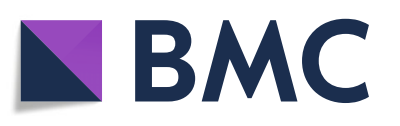

(c) The Author(s). 2021 Open Access This article is licensed under a Creative Commons Attribution 4.0 International License, which permits use, sharing, adaptation, distribution and reproduction in any medium or format, as long as you give appropriate credit to the original author(s) and the source, provide a link to the Creative Commons licence, and indicate if changes were made. The images or other third party material in this article are included in the article's Creative Commons licence, unless indicated otherwise in a credit line to the material. If material is not included in the article's Creative Commons licence and your intended use is not permitted by statutory regulation or exceeds the permitted use, you will need to obtain permission directly from the copyright holder. To view a copy of this licence, visit http://creativecommons.org/licenses/by/4.0/. The Creative Commons Public Domain Dedication waiver (http://creativecommons.org/publicdomain/zero/1.0/) applies to the data made available in this article, unless otherwise stated in a credit line to the data. 


\section{Introduction}

The global pandemic of SARS-CoV-2 infection with resulting coronavirus disease 2019 (COVID-19) has resulted in worldwide devastating health crises [1, 2]. Hemostatic abnormalities have been seen in patients with COVID-19, including elevated D-dimer levels, thrombocytopenia, increased fibrin degradation products, disseminated intravascular coagulation (DIC), and thromboembolism [1, 3-9]. The reported incidence of thromboembolic events can be $20-30 \%$ in various retrospective studies [10,11]. Furthermore, these abnormalities were associated with higher rates of mechanical ventilation, intensive care unit (ICU) admission, and death [5].

During the early phase of the COVID-19 pandemic, and with evolving knowledge about related coagulopathies and thrombosis complications, there was a lack of consensus about appropriate anticoagulation (AC) intensity. Initially, prophylactic intensity of AC was recommended for all patients hospitalized for COVID-19 [12]. However, increasing evidence demonstrated that patients developed venous thromboembolism (VTE) despite the standard pharmacological prophylaxis, especially in critically ill patients $[11,13]$. Therefore, empiric use of higher intensity therapeutic AC was proposed for patients with elevated D-dimer values, even without confirmed thromboembolism [11, 14-16]. However, safety concerns arose with evidence of increased bleeding risk in certain patients, thus there appears to be an unclear benefit with empiric therapeutic AC in COVID-19 patients with high pro-thrombotic risks $[17,18]$.

As New York City (NYC) became the epicenter of the COVID-19 pandemic in March 2020, our hospital in the South Bronx admitted and managed SARS-CoV-2 infections ranging from moderate to severe and critical disease. Therapeutic AC was used empirically at the discretion of the treating physicians when there was a high clinical suspicion of thrombosis or embolism with elevated D-dimers. We aimed at assessing the impact of empiric use of therapeutic AC on the outcome of a large cohort of hospitalized patients with COVID-19.

\section{Methods}

\section{Study design and patient population}

This is a retrospective cohort study conducted in a tertiary, acute care hospital in South Bronx, New York. We included all hospitalized adults with a positive SARSCoV-2 PCR result from March 5 to May 15, 2020. Patients who were excluded from the study were those who died or were discharged within $48 \mathrm{~h}$ after admission, those who were transferred to another facility, those who were on long-term AC before the admission, and those who received thrombolytics during the hospital course. Follow-up continued through July 1, 2020, when all participants had confirmed outcomes (discharged alive versus death). This work was approved by our hospital Institutional Review Board (IRB: 20-017).

Therapeutic anticoagulants included enoxaparin (1 $\mathrm{mg} / \mathrm{kg}$ twice daily), apixaban ( $\geq 5 \mathrm{mg}$ twice daily), unfractionated heparin (UFH) infusion, and fondaparinux ( $\geq 5 \mathrm{mg}$ once daily). The indications of empiric therapeutic AC were persistently high oxygen requirement with elevated D-dimer more than 6 times the upper limit of normal or age-adjusted D-dimer cutoff (age in years $\mathrm{x} 10 \mathrm{mcg} / \mathrm{L}$ for patients over 50 years old) [19]. Those who received therapeutic AC for known indications, including confirmed VTE (pulmonary embolism $[\mathrm{PE}]$, deep venous thrombosis [DVT]), arterial thrombotic events (acute coronary syndrome, acute ischemic stroke, and acute peripheral artery thrombosis), and atrial fibrillation, were excluded from the final analysis.

\section{Data collection and definition of variables}

Sociodemographic and broad characteristics including age, sex, ethnicity, and comorbidities (hypertension, diabetes, chronic lung disease, chronic kidney disease, congestive heart disease, chronic liver disease, cancer, HIV, transplant, and other immunosuppression conditions) and presenting symptoms were collected from medical records. The Charlson Comorbidity Index (CCI), was used as a measure of aggregate comorbidity burden [20]. Sepsis was determined by a quick SOFA score (altered mental status, respiratory rate $\geq 22$, and/or systolic blood pressure $\leq 100) \geq 2$ on admission [21]. The severity of COVID-19 infection on admission was classified into three categories (moderate, severe, critical) according to World Health Organization (WHO) guidelines. Moderate cases were defined as hospitalized patients with any of the following: fever, cough, or shortness of breath with radiographic evidence of pulmonary infiltrates and O2 saturation $>94 \%$ on room air. Severe cases were defined as hospitalized patients with respiratory rate $(R R) \geq 24 / \mathrm{min}$, O2 saturation $\leq 94 \%$, $\mathrm{PaO} 2 / \mathrm{FiO} 2<300$, and/or lung infiltrates involving > $50 \%$ of lung fields within $24-48 \mathrm{~h}$. Critical cases were defined as respiratory failure requiring mechanical ventilation, shock, or organ failure [22]. Bleeding risk was evaluated by HAS-BLED score including Hypertension, Abnormal renal/liver function, Stroke, Bleeding, Labile International Normalized Ratio (INR), Elderly, Drugs or alcohol use [23]. Major bleeding was defined as a fall in hemoglobin of $2 \mathrm{~g} /$ $\mathrm{dL}$ or more, requirement of 2 or more units of red blood cell transfusion, or any observed bleeding including intracranial hemorrhage, melena, hematemesis, hemoptysis, hematuria, etc. [24]. 


\section{Statistical analysis}

Intergroup comparison of categorical variables was performed by Pearson's Chi-square or the Fisher Exact test. Mann-Whitney U test was used to compare nonparametric continuous variables. To reduce potential confounders, we performed propensity score matching (PSM) using a 1:2 ratio with the nearest neighbor matching procedure and a caliber of 0.3 without replacement [25]. Baseline characteristics, initial symptoms, laboratory and radiographic findings, other medications, as well as the assessment of COVID-19 severity, sepsis, and acute kidney injury, were adjusted by PSM (Table 1). Adequate matching for the major imbalance of each covariate was fully assessed by visually comparing the distribution of propensity score and standardized difference (Figure S1). Univariate and multivariate cox proportional regression models were adopted to explore predictors of survival expressed as hazard ratio (HR) and 95\% confidence interval (CI). To minimize immortal bias, empiric therapeutic AC was defined as a time-dependent covariate, adjusted by time to the start of exposure. KaplanMeier curves were plotted for matched patients with therapeutic AC versus prophylactic AC. Logistic regression analysis was used to determine the association between AC intensity and mortality stratified by D-dimer, as well as between HAS-BLED score and risk of major bleeding. $P<0.05$ was considered statistically significant. All data analysis was conducted using SPSS for Windows, version 22.0 (IBM), and R software, version 3.6.1 (R Project for Statistical Computing).

\section{Results}

\section{Baseline characteristics and PSM}

Of the 1189 consecutive patients hospitalized with confirmed COVID-19 infection, 973 patients were included in the study. 209 (21.5\%) patients received therapeutic $\mathrm{AC}$ and 764 (78.5\%) received prophylactic $\mathrm{AC}$. In the therapeutic AC group, 165 individuals empirically received full dose $\mathrm{AC}$, of which 44 received higher intensity AC for specific indications (such as PE, etc., as mentioned above) and were excluded from our final analysis. A total of 28 (2.9\%) thromboembolic events were confirmed including 13 incidents of acute PE, 11 occurrences of DVT, and two individuals suffered from acute ischemic strokes, and 2 experienced acute peripheral artery occlusions (Fig. 1). The median duration of $\mathrm{AC}$ treatment was 5 days (IQR: 3-8 days). And the median time from hospital admission to the start of $\mathrm{AC}$ was 3 days (IQR: $0-5$ days). $76.3 \%$ of patients of those treated with therapeutic AC received the first dose within $72 \mathrm{~h}$ after presentation to the emergency room.

Table 1 demonstrated baseline cohort characteristics. Prior to PSM, empiric AC exposure differed between age groups, comorbidities, initial symptoms, laboratory, and radiographic findings. Patients in the therapeutic AC group had a higher prevalence of severe and critical COVID-19 and higher levels of inflammatory markers on admission. After PSM, 133 patients were included in the empiric therapeutic AC cohort while 215 patients were in the prophylactic AC cohort. All covariates were balanced between the two groups after PSM, supported by closed standard mean differences (Figure S2).

\section{Primary outcome and risk factor analysis}

Prior to PSM stratification, 83 of 165 (50.3\%) patients treated with therapeutic AC died, with a median hospital stay of 11 days compared to 165 of 764 (21.6\%) deaths and a median hospital stay of 7 days in patients receiving prophylactic AC. After PSM stratification, no statistical difference between the two groups was found when assessing for rates of invasive mechanical ventilation $(73.7 \%$ versus $65.6 \%, p=0.133)$ and mortality $(60.2 \%$ versus $60.9 \%, p=0.885)$. Also, the median duration of hospitalization was prolonged in patients treated with therapeutic AC (9 days versus 7 days, $p<0.001)$. Yet, patients receiving therapeutic AC showed a much higher risk of major bleeding $(13.8 \%$ vs $3.9 \%, p<0.001$ ) (Table 1 ).

Among patients requiring invasive mechanical ventilation, those receiving empiric therapeutic AC showed lower mortality compared to those receiving prophylactic AC only $(75.5 \%$ vs $83.7 \%, p<0.001)$. After adjusting for baseline characteristics on admission, therapeutic AC remained an independent predictor of improved survival (adjusted hazard ratio [aHR] 0.476, 95\% confidence interval [CI] 0.345-0.657, $p<0.001$ ) among intubated patients. However, empiric therapeutic AC had no significant correlation to mortality $(p=0.063)$ when evaluating the entire cohort. Besides, advanced age, critical COVID-19 infection, and sepsis on admission appeared to be independent predictors of all-cause mortality regardless of invasive mechanical ventilation (Table 2). Figure 2a displayed the Kaplan-Meier survival curve for all the patients. There was no significant difference in the median survival between the two AC cohorts ( $p=$ 0.056). As indicated in Fig. $2 \mathrm{~b}$ with a Kaplan-Meier survival curve for intubated patients only, those receiving therapeutic AC had a significantly longer median survival compared to those without therapeutic AC (14 days vs 8 days, $p<0.001)$.

We then stratified all the patients into subgroups with various cutoffs of elevated D-dimer levels (500-7000 ng/ $\mathrm{mL})$. No significant difference of mortality was found between the two AC intensities in any subgroups of Ddimer scales (Table 3).

Furthermore, patients who had a HAS-BLED score of $\geq 2$, were associated with a significantly increased risk of mortality (HR 1.482, 95\% CI 1.110-1.980, $p=0.008$ ) 
Table 1 Characteristics of all hospitalized COVID-19 patients receiving empiric therapeutic AC versus prophylactic AC

\begin{tabular}{|c|c|c|c|c|c|c|}
\hline \multirow[b]{2}{*}{ Parameters } & \multicolumn{3}{|c|}{ Prior to Propensity Score Match } & \multicolumn{3}{|c|}{ After Propensity Score Match } \\
\hline & $\begin{array}{l}\text { Therapeutic AC } \\
(n=165)\end{array}$ & $\begin{array}{l}\text { Prophylactic AC } \\
(n=764)\end{array}$ & $\begin{array}{l}p \\
\text { value }\end{array}$ & $\begin{array}{l}\text { Therapeutic AC } \\
(n=133)\end{array}$ & $\begin{array}{l}\text { Prophylactic AC } \\
(n=215)\end{array}$ & $\begin{array}{l}p \\
\text { value }\end{array}$ \\
\hline Age, median (IQR) & $61(54-72)$ & $62(50-75)$ & 0.894 & $62(54-72)$ & $65(52-75)$ & 0.345 \\
\hline Age categories, n (\%) & & & $0.001^{*}$ & & & 0.064 \\
\hline 18-39 & $11(5.3)$ & $87(11.4)$ & & $6(4.5)$ & $19(8.8)$ & \\
\hline $40-59$ & 78 (37.3) & $244(31.9)$ & & $50(37.6)$ & $61(28.4)$ & \\
\hline $60-79$ & $101(48.3)$ & $309(40.4)$ & & $65(48.9)$ & $101(47.0)$ & \\
\hline$\geq 80$ & $19(9.1)$ & $124(16.2)$ & & $12(9.0)$ & $34(15.8)$ & \\
\hline Gender, Female, n (\%) & $77(36.8)$ & $336(44.0)$ & 0.064 & $47(35.3)$ & $80(37.2)$ & 0.725 \\
\hline Ethnicity, n (\%) & & & 0.348 & & & 0.737 \\
\hline Latinx & $141(67.5)$ & $482(63.0)$ & & $97(72.9)$ & $154(71.6)$ & \\
\hline Black & $62(29.7)$ & $236(31.0)$ & & $34(25.6)$ & $55(25.6)$ & \\
\hline White & $4(1.9)$ & $29(3.8)$ & & $2(1.5)$ & $6(2.8)$ & \\
\hline Asian & $2(1.0)$ & $17(2.2)$ & & 0 & 0 & \\
\hline Body Mass Index, median (IQR) & $30.1(26.2-35.0)$ & $29.0(25.0-33.7)$ & 0.066 & $30.2(26.4-35.0)$ & $29.4(25.0-34.2)$ & 0.155 \\
\hline Body Mass Index categories, n (\%) & & & 0.136 & & & 0.258 \\
\hline Underweight & $1(0.6)$ & $14(1.8)$ & & $1(0.8)$ & $5(2.3)$ & \\
\hline Normal & $28(17.0)$ & $170(22.3)$ & & $21(15.8)$ & $42(19.1)$ & \\
\hline Overweight & $49(29.7)$ & $244(31.9)$ & & $39(29.3)$ & $74(34.4)$ & \\
\hline Obesity & $107(51.2)$ & $327(42.8)$ & & $72(54.1)$ & $95(44.2)$ & \\
\hline Current smoker, n (\%) & $4(1.9)$ & $36(4.7)$ & 0.071 & $1(0.8)$ & $3(1.4)$ & 1.000 \\
\hline \multicolumn{7}{|l|}{ Past diagnosis, n (\%) } \\
\hline Diabetes mellitus & $94(45.0)$ & $358(46.9)$ & 0.629 & $55(41.4)$ & $91(42.3)$ & 0.858 \\
\hline Hypertension & $120(57.4)$ & $322(42.1)$ & $\begin{array}{l}< \\
0.001^{*}\end{array}$ & $67(50.4)$ & $102(47.4)$ & 0.595 \\
\hline Chronic lung disease & $34(16.3)$ & $148(19.4)$ & 0.308 & $24(18.0)$ & $39(18.1)$ & 0.982 \\
\hline Congestive heart failure & $8(3.8)$ & $55(7.2)$ & $0.049^{*}$ & $6(4.5)$ & $12(5.6)$ & 0.661 \\
\hline Chronic kidney disease, stage > 3 & $24(11.5)$ & $105(13.7)$ & 0.393 & $12(9.0)$ & $23(10.7)$ & 0.614 \\
\hline Cancer & $19(9.1)$ & $52(6.8)$ & 0.260 & $10(7.5)$ & $19(8.8)$ & 0.665 \\
\hline Chronic liver disease and Cirrhosis & $9(5.5)$ & $20(2.6)$ & $0.047^{*}$ & $6(4.5)$ & $10(4.7)$ & 0.952 \\
\hline $\begin{array}{l}\text { HIV, transplant, and other } \\
\text { immunosuppression status }\end{array}$ & $7(4.2)$ & $34(4.5)$ & 0.906 & $7(5.3)$ & $11(5.1)$ & 0.952 \\
\hline $\begin{array}{l}\text { Charlson Comorbidities Index (CCI), } \\
\text { median (IQR) }\end{array}$ & $3(1-4)$ & $3(1-5)$ & 0.909 & $2(1-4)$ & $3(1-4)$ & 0.976 \\
\hline HAS-BLED score, median (IQR) & $2(1-3)$ & $2(1-4)$ & 0.061 & $2(1-2)$ & $2(1-3)$ & 0.109 \\
\hline \multicolumn{7}{|l|}{ Initial symptoms, n (\%) } \\
\hline Fever & $127(60.8)$ & $461(60.3)$ & 0.911 & $83(62.4)$ & $129(60.0)$ & 0.655 \\
\hline Cough & $148(70.8)$ & $497(65.1)$ & 0.118 & $97(72.9)$ & $149(69.3)$ & 0.470 \\
\hline Dyspnea & $166(79.4)$ & $520(68.1)$ & $0.001^{*}$ & $107(80.5)$ & $167(77.7)$ & 0.538 \\
\hline Gastrointestinal symptoms & $33(15.8)$ & $205(26.8)$ & $0.001^{*}$ & $24(18.0)$ & $51(23.7)$ & 0.211 \\
\hline Neurologic symptoms & $37(17.7)$ & $132(17.3)$ & 0.885 & $22(16.5)$ & $45(20.9)$ & 0.313 \\
\hline \multicolumn{7}{|l|}{ Initial laboratory test, median (IQR) } \\
\hline $\begin{array}{l}\text { White blood cell count }\left[4.8-10.8 \times 10^{3} /\right. \\
\mu L]\end{array}$ & $10.0(7.0-13.2)$ & $7.6(5.5-10.4)$ & $\begin{array}{l}< \\
0.001^{*}\end{array}$ & $9.8(6.9-12.5)$ & $9.0(6.6-11.5)$ & 0.165 \\
\hline $\begin{array}{l}\text { Absolute lymphocyte cell count }[1.0- \\
\left.4.8 \times 10^{3} / \mu \mathrm{L}\right]\end{array}$ & $1.0(0.6-1.3)$ & $1.0(0.7-1.4)$ & $0.027^{*}$ & $0.9(0.6-1.3)$ & $0.9(0.7-1.3)$ & 0.428 \\
\hline
\end{tabular}


Table 1 Characteristics of all hospitalized COVID-19 patients receiving empiric therapeutic AC versus prophylactic AC (Continued)

\begin{tabular}{|c|c|c|c|c|c|c|}
\hline \multirow[b]{2}{*}{ Parameters } & \multicolumn{3}{|c|}{ Prior to Propensity Score Match } & \multicolumn{3}{|c|}{ After Propensity Score Match } \\
\hline & $\begin{array}{l}\text { Therapeutic AC } \\
(n=165)\end{array}$ & $\begin{array}{l}\text { Prophylactic AC } \\
(n=764)\end{array}$ & $\begin{array}{l}p \\
\text { value }\end{array}$ & $\begin{array}{l}\text { Therapeutic AC } \\
(n=133)\end{array}$ & $\begin{array}{l}\text { Prophylactic AC } \\
(n=215)\end{array}$ & $\begin{array}{l}p \\
\text { value }\end{array}$ \\
\hline $\begin{array}{l}\text { Absolute neutrophil cell count }[1.8-7.8 \times \\
\left.10^{3} / \mu \mathrm{L}\right]\end{array}$ & $8.5(5.6-11.3)$ & $5.7(3.9-8.4)$ & $\begin{array}{l}<< \\
0.001^{*}\end{array}$ & $8.4(5.3-10.6)$ & $7.2(4.9-9.5)$ & 0.077 \\
\hline Hemoglobin [14-18 g/dL] & $13.2(11.9-14.3)$ & $13.2(11.6-14.5)$ & 0.864 & $13.0(11.6-14.1)$ & $13.1(11.5-14.6)$ & 0.467 \\
\hline 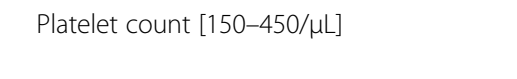 & $248.0(194.0-312.0)$ & $215.0(167.0-275.0)$ & $\begin{array}{l}< \\
0.001^{*}\end{array}$ & $241.0(192.5-307.5)$ & $232.0(177.0-310.0)$ & 0.462 \\
\hline D-dimer $[<=230$ ng/mL $]$ & $\begin{array}{l}1207.0(413.0- \\
5721.0)\end{array}$ & $498.0(258.0-897.0)$ & $\begin{array}{l}< \\
0.001^{*}\end{array}$ & $882.0(403.0-3278.5)$ & $793.0(393.0-2024.1)$ & 0.051 \\
\hline Lactate dehydrogenase [135-225 U/L] & $592.5(457.0-768.8)$ & $457.0(333.3-640.8)$ & $\begin{array}{l}< \\
0.001^{*}\end{array}$ & $599.0(459.0-724.5)$ & $605.0(398.0-715.0)$ & 0.597 \\
\hline C-reactive protein [0-0.40 mg/dL] & $19.1(10.8-30.0)$ & $11.3(5.5-21.3)$ & $\begin{array}{l}< \\
0.001^{*}\end{array}$ & $20.7(11.2-27.1)$ & $18.3(10.8-26.5)$ & 0.368 \\
\hline Interleukin-6 [0-5.5 pg/mL] & $129.5(62.1-285.8)$ & $78.3(27.1-172.3)$ & $\begin{array}{l}< \\
0.001^{*}\end{array}$ & $221.7(92.6-299.5)$ & $238.6(96.7-278.7)$ & 0.249 \\
\hline Ferritin [12-300 ng/mL] & $883.0(492.0-1606.0)$ & $688.0(365.0-1224.5)$ & $0.002^{*}$ & $\begin{array}{l}1070.0(502.5- \\
1532.5)\end{array}$ & $\begin{array}{l}1067.0(562.0- \\
1387.5)\end{array}$ & 0.568 \\
\hline \multicolumn{7}{|l|}{ Radiographic findings, n (\%) } \\
\hline Bilateral patchy infiltrates & $131(62.7)$ & $412(53.9)$ & $0.024^{*}$ & $91(68.4)$ & $129(60.0)$ & 0.137 \\
\hline Ground glass opacity & $82(39.2)$ & $217(28.4)$ & $0.003^{*}$ & $53(39.8)$ & $70(32.6)$ & 0.238 \\
\hline Consolidation & $19(9.1)$ & $102(13.4)$ & 0.098 & $12(9.0)$ & $26(12.1)$ & 0.207 \\
\hline Atelectasis & $4(1.9)$ & $33(4.3)$ & 0.107 & $2(1.5)$ & $7(3.3)$ & 0.491 \\
\hline \multicolumn{7}{|l|}{ Other medications received, n (\%) } \\
\hline Hydroxychloroquine & $167(79.9)$ & $542(70.9)$ & $0.010^{*}$ & $105(78.9)$ & $165(76.6)$ & 0.589 \\
\hline Azithromycin & $177(84.7)$ & $553(72.4)$ & $\begin{array}{l}< \\
0.001^{*}\end{array}$ & $111(83.4)$ & $173(80.6)$ & 0.480 \\
\hline Remdesevir & $15(7.2)$ & $1(0.1)$ & $\begin{array}{l}< \\
0.001^{*}\end{array}$ & $1(0.8)$ & $1(0.5)$ & 0.555 \\
\hline Sarilumab/Tocilizumab & $26(12.4)$ & $35(4.6)$ & $\begin{array}{l}< \\
0.001^{*}\end{array}$ & $15(11.3)$ & $20(9.4)$ & 0.540 \\
\hline Convalescent plasma & $22(10.5)$ & $6(0.8)$ & $\begin{array}{l}< \\
0.001^{*}\end{array}$ & $7(5.3)$ & $6(3.0)$ & 0.122 \\
\hline Stress dose of steroid & $55(33.3)$ & $74(9.7)$ & $\begin{array}{l}< \\
0.001^{*}\end{array}$ & 28 (18.6) & $40(18.4)$ & 0.756 \\
\hline $\begin{array}{l}\text { COVID-19 severity classification on } \\
\text { admission, } \mathrm{n}(\%)\end{array}$ & & & $<.001^{*}$ & & & 0.458 \\
\hline Moderate & $21(10.0)$ & $378(49.3)$ & & $13(9.8)$ & $32(14.9)$ & \\
\hline Severe & $83(39.7)$ & $209(27.4)$ & & $47(35.3)$ & $80(37.2)$ & \\
\hline Critical & $105(50.2)$ & $177(23.2)$ & & $73(54.9)$ & $103(47.9)$ & \\
\hline Sepsis by quick SOFA on admission, $\mathrm{n}(\%)$ & $37(22.4)$ & $230(30.1)$ & $0.048^{*}$ & $35(26.3)$ & $51(23.7)$ & 0.586 \\
\hline Acute kidney injury on admission, n (\%) & $53(32.1)$ & $218(28.5)$ & 0.358 & 45 (33.8) & 77 (35.8) & 0.707 \\
\hline \multicolumn{7}{|l|}{ Hospital course (unmatched) } \\
\hline Invasive mechanical ventilation, n (\%) & $128(77.6)$ & $221(28.9)$ & $\begin{array}{l}< \\
0.001^{*}\end{array}$ & $98(73.7)$ & $141(65.6)$ & 0.113 \\
\hline Major bleeding, n (\%) & $23(14.1)$ & $27(3.5)$ & $\begin{array}{l}< \\
0.001^{*}\end{array}$ & $18(13.8)$ & $8(3.9)$ & $\begin{array}{l}< \\
0.001^{*}\end{array}$ \\
\hline Days of hospitalization, median (IQR) & $11(6-21)$ & $7(4-12)$ & $\begin{array}{l}< \\
0.001^{*}\end{array}$ & $9(6-19)$ & $7(5-12)$ & $<.001^{*}$ \\
\hline All-cause mortality, n (\%) & $83(50.3)$ & $165(21.6)$ & $\begin{array}{l}< \\
0.001^{*}\end{array}$ & $80(60.2)$ & $131(60.9)$ & 0.885 \\
\hline
\end{tabular}




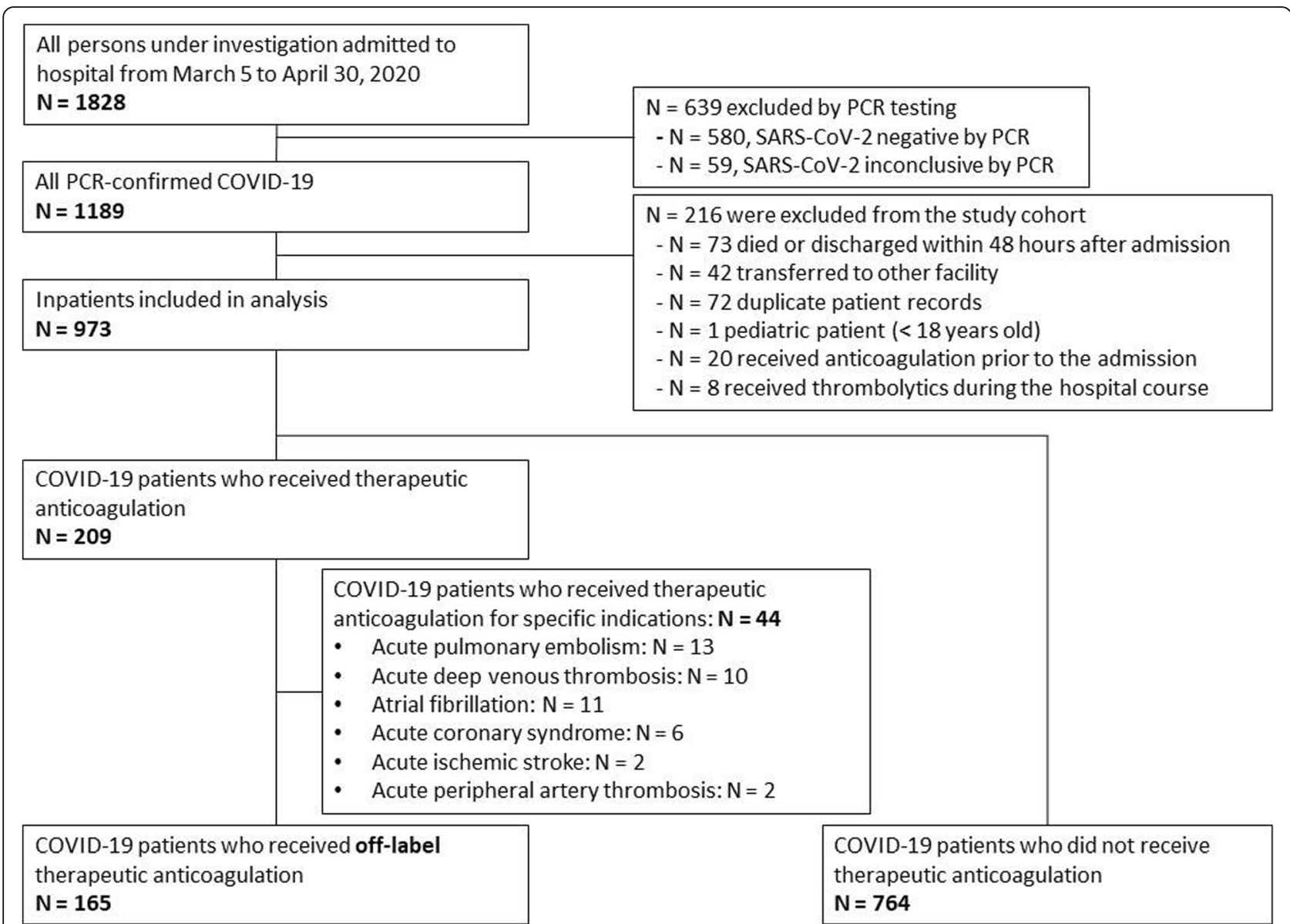

Fig. 1 Flow diagram of process for patient inclusion as participants in the study

(Table 2). Additional analysis showed that a high HASBLED score $(\geq 3)$ was associated with a significantly likelihood of major bleeding (incidence of major bleeding: $11.4 \%$ vs $6.1 \%$; Odds ratio: 1.883 , CI: $1.114-3.729, p=$ 0.016) (Table 4).

\section{Discussion}

Our analysis suggested that empiric use of therapeutic $\mathrm{AC}$ in patients requiring invasive mechanical ventilation significantly improved survival after adjustment of age, gender, race, comorbidities, COVID-19 disease severity, and major complications. However, our results did not demonstrate any survival benefit with therapeutic AC in non-critically ill patients. Lemos et al. reported similar results in a randomized clinical trial where the use of therapeutic intensity enoxaparin resulted in improved gas exchange over time, decreased D-dimer levels, and subsequently higher rates of successful liberation from mechanical ventilation after respiratory failure in severe COVID-19 patients [26]. The trial only included mechanically ventilated patients with $\mathrm{D}$-dimer concentration greater than $1000 \mu \mathrm{g} / \mathrm{L}$, no older than 85 years-old without severe organ dysfunction prior COVID-19 infection (ESRD, Child B or C cirrhoris, Heart failure class III or IV, etc.) or patients with contraindication for full AC [26] (Table S1). In contrast, the American Society of Hematology guidelines panel suggested use of prophylactic intensity of $\mathrm{AC}$ over intermediate or therapeutic intensity of AC in COVID-19 related critical illness without suspected or confirmed VTE [12], but these recommendations are based on limited evidence. Tremblay et al. compared patients on AC prior to COVID-19 infection with patients without AC or antiplalete therapy, and matched them by propensity score method. The factor of AC did not reduce the risk for all-cause of mortality, mechanical ventilation or hospital admission [27]. Still, despite widespread use of therapeutic AC, there is a current paucity of data to support empiric use in COVID-19 patients with high pro-thrombotic risk. Thus, our findings may contribute towards the ever-growing body of evidence regarding AC use in COVID-19 disease and may further contribute to therapeutic guidance.

The precise mechanistic advantage of AC remains unclear. AC was presumed to provide benefit by partially 
Table 2 Cox proportional hazards model of risk factors for all-cause mortality among patients with or without receiving empiric therapeutic AC

\begin{tabular}{|c|c|c|c|c|c|c|c|c|c|c|}
\hline & \multicolumn{5}{|c|}{ All matched patients } & \multicolumn{5}{|c|}{ Patients requiring invasive mechanical ventilation } \\
\hline & \multirow[t]{2}{*}{$\begin{array}{l}\text { Death/N at risk } \\
\text { (\%) }\end{array}$} & \multicolumn{2}{|c|}{ Univariate analysis } & \multicolumn{2}{|c|}{$\begin{array}{l}\text { Multivariate } \\
\text { analysis }\end{array}$} & \multirow[t]{2}{*}{$\begin{array}{l}\text { Death/N at risk } \\
\text { (\%) }\end{array}$} & \multicolumn{2}{|c|}{ Univariate analysis } & \multicolumn{2}{|c|}{$\begin{array}{l}\text { Multivariate } \\
\text { analysis }\end{array}$} \\
\hline & & $\begin{array}{l}\mathrm{HR}(95 \% \\
\mathrm{Cl})\end{array}$ & $\begin{array}{l}p \\
\text { value }\end{array}$ & $\begin{array}{l}\mathrm{HR}(95 \% \\
\mathrm{Cl})\end{array}$ & $\begin{array}{l}p \\
\text { value }\end{array}$ & & $\begin{array}{l}\mathrm{HR}(95 \% \\
\mathrm{Cl})\end{array}$ & $\begin{array}{l}p \\
\text { value }\end{array}$ & $\begin{array}{l}\text { HR }(95 \% \\
\mathrm{Cl})\end{array}$ & $\begin{array}{l}p \\
\text { value }\end{array}$ \\
\hline \multicolumn{11}{|c|}{ Empiric therapeutic AC } \\
\hline No & $131 / 215(60.9)$ & Reference & & & & $118 / 141(83.7)$ & Reference & & Reference & \\
\hline Yes & $80 / 133(60.2)$ & $\begin{array}{l}0.792 \\
(0.619- \\
1.013)\end{array}$ & 0.063 & & & $74 / 98(75.5)$ & $\begin{array}{l}0.536 \\
(0.413- \\
0.695)\end{array}$ & <. $0001^{*}$ & $\begin{array}{l}0.476 \\
(0.345- \\
0.657)\end{array}$ & $\begin{array}{l}< \\
0.001^{*}\end{array}$ \\
\hline \multicolumn{11}{|l|}{ Age } \\
\hline$<65$ years old & 103/199 (51.8) & Reference & & Reference & & $98 / 138(71.0)$ & Reference & & Reference & \\
\hline$\geq 65$ years old & $108 / 149(72.5)$ & $\begin{array}{l}1.423 \\
(1.190- \\
1.701)\end{array}$ & $\begin{array}{l}< \\
0.001^{*}\end{array}$ & $\begin{array}{l}1.028 \\
(1.014- \\
1.142)\end{array}$ & $\begin{array}{l}< \\
0.001^{*}\end{array}$ & 90/101 (89.1) & $\begin{array}{l}1.276 \\
(1.042- \\
1.563)\end{array}$ & $0.008^{*}$ & $\begin{array}{l}1.207 \\
(1.006- \\
1.526)\end{array}$ & $0.015^{*}$ \\
\hline \multicolumn{11}{|l|}{ Gender } \\
\hline Male & $133 / 221(60.2)$ & Reference & & & & 119/151 (78.8) & Reference & & & \\
\hline Female & $78 / 127(61.4)$ & $\begin{array}{l}0.915 \\
(0.692- \\
1.211)\end{array}$ & 0.536 & & & $73 / 88(83.0)$ & $\begin{array}{l}0.920 \\
(0.687- \\
1.232)\end{array}$ & 0.577 & & \\
\hline \multicolumn{11}{|l|}{ Ethnicity } \\
\hline Non-Latinx & $56 / 97(57.7)$ & Reference & & & & $51 / 64(79.7)$ & Reference & & & \\
\hline Latinx & 155/251 (61.8) & $\begin{array}{l}0.930 \\
(0.709- \\
1.221)\end{array}$ & 0.603 & & & 141/175 (80.6) & $\begin{array}{l}1.082 \\
(0.806- \\
1.453)\end{array}$ & 0.598 & & \\
\hline \multicolumn{11}{|l|}{ Body-mass index } \\
\hline$<30$ & 104/181 (57.4) & Reference & & & & $87 / 111(78.4)$ & Reference & & & \\
\hline$\geq 30$ & 107/167 (64.1) & $\begin{array}{l}1.011 \\
(0.995- \\
1.027)\end{array}$ & 0.188 & & & 105/128 (82.0) & $\begin{array}{l}1.002 \\
(0.986- \\
1.018)\end{array}$ & 0.818 & & \\
\hline \multicolumn{11}{|l|}{ Comorbidities } \\
\hline $\begin{array}{l}\text { Diabetes } \\
\text { mellitus }\end{array}$ & $91 / 146(62.3)$ & $\begin{array}{l}1.087 \\
(0.828- \\
1.428)\end{array}$ & 0.548 & & & $81 / 102(79.4)$ & $\begin{array}{l}0.996 \\
(0.748- \\
1.326)\end{array}$ & 0.975 & & \\
\hline Hypertension & $105 / 168(62.1)$ & $\begin{array}{l}1.126 \\
(0.860- \\
1.475)\end{array}$ & 0.389 & & & 89/106 (84.0) & $\begin{array}{l}1.214 \\
(0.914- \\
1.613)\end{array}$ & 0.180 & & \\
\hline COPD/asthma & 45/63 (71.4) & $\begin{array}{l}1.429 \\
(1.028- \\
1.988)\end{array}$ & $0.034^{*}$ & & & 45/50 (90.0) & $\begin{array}{l}1.502 \\
(1.074- \\
2.101)\end{array}$ & $0.017^{*}$ & & \\
\hline $\begin{array}{l}\text { Congestive } \\
\text { heart failure }\end{array}$ & $11 / 18(61.1)$ & $\begin{array}{l}1.196 \\
(0.652- \\
2.195)\end{array}$ & 0.564 & & & 10/11 (90.9) & $\begin{array}{l}1.196 \\
(1.067- \\
3.846)\end{array}$ & $0.031^{*}$ & & \\
\hline $\begin{array}{l}\text { Chronic kidney } \\
\text { disease }\end{array}$ & 23/35 (65.7) & $\begin{array}{l}1.196 \\
(0.775- \\
1.843)\end{array}$ & 0.419 & & & $17 / 22(77.3)$ & $\begin{array}{l}0.899 \\
(0.547- \\
1.480)\end{array}$ & 0.676 & & \\
\hline Cancer & 21/29 (72.4) & $\begin{array}{l}1.341 \\
(0.854- \\
2.106)\end{array}$ & 0.202 & & & 16/19 (84.2) & $\begin{array}{l}1.000 \\
(0.599- \\
1.668)\end{array}$ & 1.000 & & \\
\hline \multicolumn{11}{|l|}{$\mathrm{CCl}$} \\
\hline$<2$ & $66 / 120(55.0)$ & Reference & & & & 65/84 (77.4) & Reference & & & \\
\hline$\geq 2$ & $145 / 228(63.6)$ & $\begin{array}{l}1.469 \\
(1.000- \\
2.799)\end{array}$ & 0.050 & & & 127/155 (81.9) & $\begin{array}{l}1.411 \\
(0.908- \\
2.931)\end{array}$ & 0.091 & & \\
\hline
\end{tabular}


Table 2 Cox proportional hazards model of risk factors for all-cause mortality among patients with or without receiving empiric therapeutic AC (Continued)

\begin{tabular}{|c|c|c|c|c|c|c|c|c|c|c|}
\hline & \multicolumn{5}{|c|}{ All matched patients } & \multicolumn{5}{|c|}{ Patients requiring invasive mechanical ventilation } \\
\hline & \multirow[t]{2}{*}{$\begin{array}{l}\text { Death/N at risk } \\
\text { (\%) }\end{array}$} & \multicolumn{2}{|c|}{ Univariate analysis } & \multicolumn{2}{|c|}{$\begin{array}{l}\text { Multivariate } \\
\text { analysis }\end{array}$} & \multirow[t]{2}{*}{$\begin{array}{l}\text { Death/N at risk } \\
\text { (\%) }\end{array}$} & \multicolumn{2}{|c|}{ Univariate analysis } & \multicolumn{2}{|c|}{$\begin{array}{l}\text { Multivariate } \\
\text { analysis }\end{array}$} \\
\hline & & $\begin{array}{l}\text { HR }(95 \% \\
\mathrm{Cl})\end{array}$ & $\begin{array}{l}p \\
\text { value }\end{array}$ & $\begin{array}{l}\mathrm{HR}(95 \% \\
\mathrm{Cl})\end{array}$ & $\begin{array}{l}p \\
\text { value }\end{array}$ & & $\begin{array}{l}\text { HR }(95 \% \\
\mathrm{Cl})\end{array}$ & $\begin{array}{l}p \\
\text { value }\end{array}$ & $\begin{array}{l}\mathrm{HR}(95 \% \\
\mathrm{Cl})\end{array}$ & $\begin{array}{l}p \\
\text { value }\end{array}$ \\
\hline \multicolumn{11}{|c|}{ COVID-19 severity } \\
\hline Moderate & $8 / 45(17.8)$ & Reference & & Reference & & 4/9 (44.4) & Reference & & Reference & \\
\hline Severe & $61 / 127(48.0)$ & $\begin{array}{l}2.282 \\
(1.091- \\
4771)\end{array}$ & $0.028^{*}$ & $\begin{array}{l}2.319 \\
(1.092- \\
4.924)\end{array}$ & $0.029^{*}$ & $45 / 61(73.7)$ & $\begin{array}{l}2.442 \\
(0.882- \\
6.765)\end{array}$ & 0.086 & & \\
\hline Critical & $142 / 176(80.7)$ & $\begin{array}{l}3.242 \\
(1.588- \\
6.620)\end{array}$ & $0.001^{*}$ & $\begin{array}{l}3.537 \\
(1.706- \\
7.330)\end{array}$ & $0.001^{*}$ & $143 / 169(84.6)$ & $\begin{array}{l}2.810 \\
(1.011- \\
6.921)\end{array}$ & $0.033^{*}$ & $\begin{array}{l}2.120 \\
(1.004- \\
6.715)\end{array}$ & $0.025^{*}$ \\
\hline \multicolumn{11}{|l|}{ Sepsis } \\
\hline No & $143 / 262(54.6)$ & Reference & & Reference & & $130 / 170(76.5)$ & Reference & & Reference & \\
\hline Yes & $68 / 86(79.1)$ & $\begin{array}{l}2.456 \\
(1.675- \\
3.602)\end{array}$ & $\begin{array}{l}< \\
0.001^{*}\end{array}$ & $\begin{array}{l}1.354 \\
(1.005- \\
1.795)\end{array}$ & $0.043^{*}$ & $62 / 69(89.9)$ & $\begin{array}{l}1.531 \\
(1.160- \\
2.764)\end{array}$ & $0.012^{*}$ & $\begin{array}{l}1.414 \\
(1.012- \\
2.705)\end{array}$ & $0.032^{*}$ \\
\hline \multicolumn{11}{|c|}{ Acute kidney injury } \\
\hline No & $128 / 226(56.6)$ & Reference & & & & 117/152 (77.0) & Reference & & & \\
\hline Yes & $83 / 122(68.0)$ & $\begin{array}{l}1.367 \\
(1.037- \\
1.802)\end{array}$ & $0.027^{*}$ & & & 75/87 (86.2) & $\begin{array}{l}1.463 \\
(0.952- \\
2.247)\end{array}$ & 0.083 & & \\
\hline \multicolumn{11}{|c|}{ HAS-BLED score } \\
\hline$\geq 1$ vs $<1$ & $\begin{array}{l}190 / 307(61.9) \text { vs } \\
21 / 41(51.2)\end{array}$ & $\begin{array}{l}1.267 \\
(0.942- \\
1.693)\end{array}$ & 0.119 & & & $\begin{array}{l}171 / 212(80.7) \text { vs } \\
21 / 27(77.8)\end{array}$ & $\begin{array}{l}1.203 \\
(0.762- \\
1.898)\end{array}$ & 0.427 & & \\
\hline$\geq 2$ vs $<2$ & $\begin{array}{l}143 / 218(65.9) \text { vs } \\
68 / 130(52.3)\end{array}$ & $\begin{array}{l}1.482 \\
(1.110- \\
1.980)\end{array}$ & $0.008^{*}$ & & & $\begin{array}{l}125 / 153(81.7) \text { vs } \\
67 / 86(77.9)\end{array}$ & $\begin{array}{l}1.250 \\
(0.928- \\
1.684)\end{array}$ & 0.141 & & \\
\hline$\geq 3$ vs $<3$ & $\begin{array}{l}65 / 88(73.9) \text { vs } 146 / \\
260(56.2)\end{array}$ & $\begin{array}{l}1.368 \\
(1.032- \\
1.952)\end{array}$ & $0.015^{*}$ & & & $\begin{array}{l}59 / 70(84.3) \text { vs } \\
133 / 169(78.7)\end{array}$ & $\begin{array}{l}1.128 \\
(0.829- \\
1.535)\end{array}$ & 0.443 & & \\
\hline
\end{tabular}

AC anticoagulation, CCI Charlson Comorbidity Index, Cl confidence interval, COPD chronic obstructive pulmonary disease, COVID-19 coronavirus disease 2019, HAS$B L E D$ Hypertension, Abnormal renal/liver function, Stroke, Bleeding, Labile International Normalized Ratio (INR), Elderly, Drugs or alcohol use, HR hazard ratio *Significant at $p<0.05$

blocking thrombin formation and thus dampen the overwhelming inflammatory response following lung tissue damage in acute respiratory distress syndrome (ARDS) $[28,29]$. However, post-mortem studies showed that thrombi in COVID-19 often developed as a consequence of direct vascular damage associated with viral infection and severe inflammation [17]. If so, the role of therapeutic AC in this scenario might not provide greater benefit than standard prophylactic AC, yet raises bleeding risk [18]. Yet, while the therapeutic mechanism remains unclear, our findings demonstrated that therapeutic AC provided mortality benefit to ICU patients with critical COVID-19 disease, but overall, we observed increased adverse bleeding events. Therefore, since critically ill COVID-19 patients admitted to the ICU carried higher thromboembolic risks (especially if intubated, sedated, paralyzed, and immobilized) then therapeutic AC may be reasonably considered. However, while escalating $\mathrm{AC}$ intensity may improve outcomes, its use should be balanced with bleeding risks on a case-tocase basis to mitigate the hemorrhagic events.

Since AC likely carries benefits, yet brings an elevated risk of adverse bleeding, D-dimer levels were initially used as a biomarker to guide appropriate use of AC therapy. Studies have demonstrated a correlation between varying levels of D-dimer (ranging from $>1000$ $\mathrm{ng} / \mathrm{ml}$ to $>2590 \mathrm{ng} / \mathrm{ml}$ ) and PE or mortality in patients with COVID-19 $[2,10,30]$. Early data from Tang et al. suggested that patients with markedly elevated D-dimer levels $(>3000 \mathrm{ng} / \mathrm{mL}$ or $>6$-fold of upper limit normal) would likely benefit from a higher dose of $\mathrm{AC}$ even with a low risk of thromboembolism [14, 15]. In contrast, recent evidence recommended against using D-dimer level cutoffs as the sole criteria to guide the intensity of AC 
A

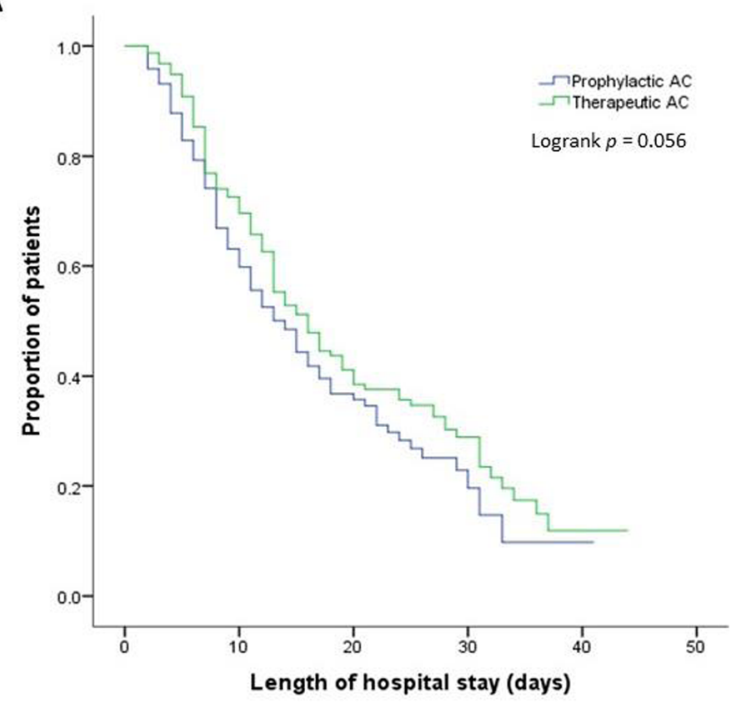

B

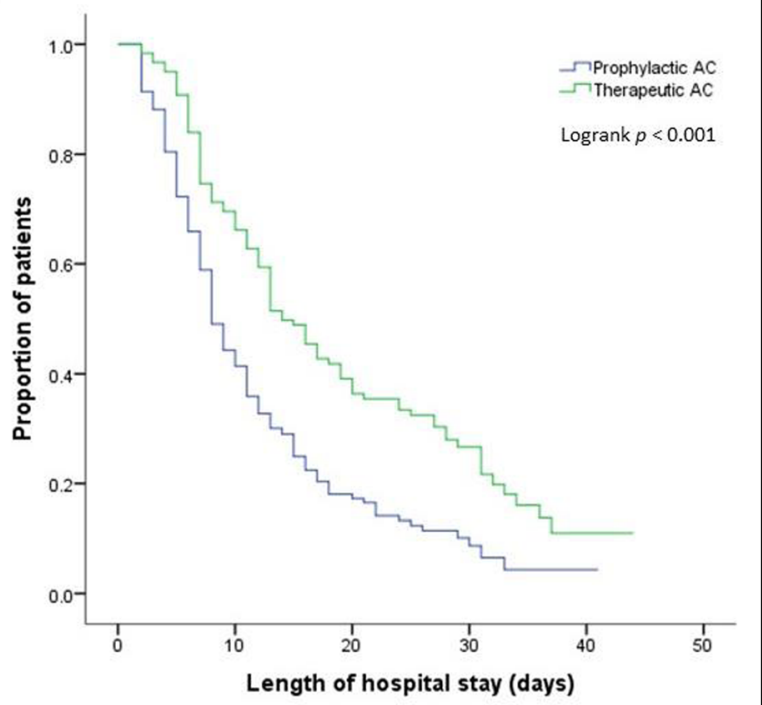

No. of patients at risk

Therapeutic AC 133

Prophylactic AC 215
$90 \quad 43$

$154 \quad 38$
8

No. of patients at risk

Fig. 2 Kaplan Meier Curve for all hospitalized patients (a) and patients requiring invasive mechanical ventilation (b) with COVID-19 according to receiving therapeutic $A C$ versus prophylactic $A C$

$[5,12]$. Our results further demonstrated that D-dimer levels did not correlate well with improved primary outcomes with empiric therapeutic versus prophylactic AC. It is noteworthy that D-dimer is a sensitive biomarker to rule out VTE, however, it has low specificity and may be elevated due to ongoing activation of the hemostatic system with inflammation, sepsis, liver disease, etc. (all of which can all be encountered in COVID-19). As such, elevated d-dimer alone is not sufficient to guide $\mathrm{AC}$ intensity, rather the whole clinical scenario should be considered.

Compared to other studies, our results showed a higher frequency of major bleeding among patients receiving therapeutic $\mathrm{AC}[18,31]$. It is likely related to the fact that only the highest doses were used in our study cohort, instead of the intermediate doses seen in other similar studies $[18,31]$. Interestingly, HAS-BLED scores may offer a promising tool to assess bleeding risk. This risk-assessment tool was initially developed to evaluate bleeding risk in anti-coagulated patients affected by atrial fibrillation [32], and later was applied for patients with acute VTE [33-35], acute coronary syndrome (ACS) [36, 37], intracranial hemorrhage (ICH) $[38,39]$, postoperative $\mathrm{AC}$ after cardiac and vascular interventions [40, 41], etc. A score of 0 indicates low risk, 1-2 indicates moderate risk, and $\geq 3$ indicates high risk.

Table 3 The association between empiric therapeutic AC and mortality of patients with COVID-19 based on the D-dimer level

\begin{tabular}{|c|c|c|c|c|c|}
\hline \multirow{2}{*}{$\begin{array}{l}\text { D- } \\
\text { Dimer } \\
\text { level } \\
\text { (ng/ } \\
\mathrm{mL} \text { ) }\end{array}$} & \multicolumn{3}{|c|}{ N. of death/N. at risk (\%) } & \multicolumn{2}{|l|}{ Univariate analysis } \\
\hline & Total & Therapeutic AC & Prophylactic AC & OR $(95 \% \mathrm{Cl})$ & $p$ value \\
\hline$\geq 500$ & $150 / 230(65.2)$ & $57 / 92(62.0)$ & $93 / 138$ (67.4) & $0.788(0.454-1.367)$ & 0.397 \\
\hline$\geq 1000$ & $89 / 134(66.4)$ & 39/63 (61.9) & 50/71 (70.4) & $0.683(0.332-1.402)$ & 0.297 \\
\hline$\geq 2000$ & 65/103 (63.1) & 29/49 (59.2) & $36 / 54(66.7)$ & $0.725(0.325-1.618)$ & 0.432 \\
\hline$\geq 3000$ & $52 / 80(65.0)$ & 23/36 (63.9) & 29/44 (65.9) & $0.915(0.364-2.302)$ & 0.851 \\
\hline$\geq 4000$ & $37 / 59(62.7)$ & $16 / 29(55.2)$ & $21 / 30(70.0)$ & $0.527(0.181-1.538)$ & 0.239 \\
\hline$\geq 5000$ & $34 / 50(68.0)$ & $16 / 26(61.5)$ & 18/24 (75.0) & $0.533(0.158-1.799)$ & 0.308 \\
\hline$\geq 6000$ & $30 / 44(68.2)$ & 13/21 (61.9) & 17/23 (73.9) & $0.574(0.159-2.066)$ & 0.393 \\
\hline$\geq 7000$ & 26/39 (66.7) & $11 / 19(57.9)$ & 15/20 (75.0) & $0.458(0.117-1.789)$ & 0.257 \\
\hline
\end{tabular}


Table 4 The association between HAS-BLED score and major bleeding

\begin{tabular}{|c|c|c|c|c|}
\hline \multirow{2}{*}{$\begin{array}{l}\text { HAS- } \\
\text { BLED } \\
\text { score } \\
\text { cutoff }\end{array}$} & \multicolumn{2}{|c|}{ Major bleeding/N. at risk (\%) } & \multicolumn{2}{|l|}{ Univariate analysis } \\
\hline & $\geq$ cutoff & $<$ cutoff & OR $(95 \% \mathrm{Cl})$ & $p$ value \\
\hline 1 & 23/307 (7.5) & $3 / 41(7.3)$ & $1.046(0.624-1.714)$ & 0.488 \\
\hline 2 & $12 / 130(9.2)$ & $14 / 218(6.4)$ & $1.256(0.832-2.114)$ & 0.103 \\
\hline 3 & 10/88 (11.4) & $16 / 260(6.1)$ & $1.883(1.114-3.729)$ & $0.016^{*}$ \\
\hline 4 & $6 / 30(20.0)$ & 20/318 (9.2) & $2.112(1.152-4.421)$ & $0.001^{*}$ \\
\hline
\end{tabular}

Our post-hoc analysis consistently demonstrated that a higher HAS-BLED score was associated with worse outcomes, with a score of $\geq 3$ correlated with a higher bleeding risk and a score of $\geq 2$ associated with a higher mortality. We suggested that use of the HAS-BLED rating system may help assess for risk stratification and guide $\mathrm{AC}$ dose selection.

Several limitations should be noted in the present study $[10,11]$. First, by nature being a single-center retrospective review, this study is limited by sample size and generalizability. Also, the decision to start therapeutic $\mathrm{AC}$ was at the discretion of the treating physician, hence we believe there might have been selection bias, which created a smaller untreated group of critically ill patients. We were unable to match for all critically ill patients receiving $\mathrm{AC}$, so there may have been residual confounding despite our use of propensity matching methods. Finally, due to COVID-19 related logistic constraints of treating COVID-19 during a time when the hospital was under surge capacity, diagnostic imaging for VTEs especially CT scans were restricted, even for critically ill and mechanically ventilated patients [14, 15].

\section{Conclusions}

Our results demonstrated that the empiric use of therapeutic AC conferred survival benefit to critically ill patients requiring invasive mechanical ventilation, but had limited benefit to non-critically ill patients hospitalized for COVID-19. Given the higher risk of mortality among patients with a high HAS-BLED score, we believe that a balanced assessment of $\mathrm{AC}$ benefits and bleeding risks is important before considering escalation of AC intensity.

\section{Supplementary Information}

The online version contains supplementary material available at https://doi. org/10.1186/s40364-021-00283-y.

\section{Additional file 1.}

\section{Acknowledgements}

This paper is dedicated to the memory of Dr. Bo Yu, whose devotion and dedication to his work is unsurpassed.

\section{Authors' contributions}

BY, VP and VM designed the study. BY, VP, AC, and AP collected all the data. BY and DK performed the statistical analysis. BY and VP drafted the manuscript. VM, DK, and GH participated in the revision of the manuscript. All authors read and approved the final manuscript.

\section{Funding}

None

\section{Availability of data and materials}

All data generated or analyzed during this study are included in the manuscript and the supplementary material.

\section{Declarations}

\section{Ethics approval and consent to participate}

This study was approved by the Institutional Review Board of New York City Health + Hospitals/ Lincoln Medical Center (IRB: 20-017).

\section{Consent for publication}

Written informed consent was waived due to urgent need of data collection.

\section{Competing interests}

The authors have no relevant conflicts.

\section{Author details}

'Department of Medicine, New York City Health + Hospitals, Lincoln Medical Center, Bronx, New York, USA. ${ }^{2}$ Department of Epidemiology and Biostatistics, University of California San Francisco, San Francisco, California, USA. ${ }^{3}$ Department of Medicine, University of California San Francisco, San Francisco, California, USA. ${ }^{4}$ Institute of Global Health Science, University of California San Francisco, San Francisco, California, USA. ${ }^{5}$ F.I. Proctor

Foundation, University of California San Francisco, San Francisco, California, USA.

Received: 14 February 2021 Accepted: 15 April 2021

Published online: 01 May 2021

\section{References}

1. Guan WJ, Ni ZY, Hu Y, Liang WH, Ou CQ, He JX, et al. Clinical characteristics of coronavirus disease 2019 in China. N Engl J Med. 2020;382(18):1708-20. https://doi.org/10.1056/NEJMoa2002032.

2. Zhou F, Yu T, Du R, Fan G, Liu Y, Liu Z, et al. Clinical course and risk factors for mortality of adult inpatients with COVID-19 in Wuhan, China: a retrospective cohort study. Lancet. 2020;395(10229):1054-62. https://doi. org/10.1016/S0140-6736(20)30566-3.

3. Zhang S, Liu Y, Wang X, Yang L, Li H, Wang Y, et al. SARS-CoV-2 binds platelet ACE2 to enhance thrombosis in COVID-19. J Hematol Oncol. 2020; 13(1):120. https://doi.org/10.1186/s13045-020-00954-7.

4. Danwang C, Endomba FT, Nkeck JR, Wouna DLA, Robert A, Noubiap JJ. A meta-analysis of potential biomarkers associated with severity of coronavirus disease 2019 (COVID-19). Biomark Res. 2020;8(1):37. https://doi. org/10.1186/s40364-020-00217-0.

5. Tang N, Li D, Wang X, Sun Z. Abnormal coagulation parameters are associated with poor prognosis in patients with novel coronavirus pneumonia. J Thromb Haemost. 2020;18(4):844-7. https://doi.org/10.1111/ jth.14768.

6. Bikdeli B, Madhavan MV, Jimenez D, Chuich T, Dreyfus I, Driggin E, et al. COVID-19 and thrombotic or thromboembolic disease: implications for prevention, antithrombotic therapy, and follow-up: JACC state-of-the-art review. J Am Coll Cardiol. 2020;75(23):2950-73. https://doi.org/10.1016/j.ja cc.2020.04.031.

7. Mei H, Luo L, Hu Y. Thrombocytopenia and thrombosis in hospitalized patients with COVID-19. J Hematol Oncol. 2020;13(1):161. https://doi.org/1 0.1186/s13045-020-01003-Z

8. Bao C, Tao X, Cui W, Yi B, Pan T, Young KH, et al. SARS-CoV-2 induced thrombocytopenia as an important biomarker significantly correlated with abnormal coagulation function, increased intravascular blood clot risk and mortality in COVID-19 patients. Exp Hematol Oncol. 2020;9(1):16. https://doi. org/10.1186/s40164-020-00172-4. 
9. Som A, Lang M, Little B. Pulmonary vascular pathology in Covid-19. N Engl J Med. 2020;383(9):887. https://doi.org/10.1056/NEJMc2022068.

10. Cui S, Chen S, Li X, Liu S, Wang F. Prevalence of venous thromboembolism in patients with severe novel coronavirus pneumonia. J Thromb Haemost. 2020;18(6):1421-4. https://doi.org/10.1111/jth.14830.

11. Klok FA, Kruip M, van der Meer NJM, Arbous MS, Gommers D, Kant KM, et al. Incidence of thrombotic complications in critically ill ICU patients with COVID-19. Thromb Res. 2020;191:145-7. https://doi.org/10.1016/j.thromres.2 020.04.013.

12. Barnes GD, Burnett A, Allen A, Blumenstein M, Clark NP, Cuker A, et al. Thromboembolism and anticoagulant therapy during the COVID-19 pandemic: interim clinical guidance from the anticoagulation forum. $J$ Thromb Thrombolysis. 2020;50(1):72-81. https://doi.org/10.1007/s11239-02002138-z.

13. Helms J, Tacquard C, Severac F, Leonard-Lorant I, Ohana M, Delabranche X, et al. High risk of thrombosis in patients with severe SARS-CoV-2 infection: a multicenter prospective cohort study. Intensive Care Med. 2020;46(6):108998. https://doi.org/10.1007/s00134-020-06062-x.

14. Atallah B, Mallah SI, AlMahmeed W. Anticoagulation in COVID-19. Eur Heart J Cardiovasc Pharmacother. 2020;6(4):260-1. https://doi.org/10.1093/ehjcvp/ pvaa036.

15. Tang N, Bai H, Chen X, Gong J, Li D, Sun Z. Anticoagulant treatment is associated with decreased mortality in severe coronavirus disease 2019 patients with coagulopathy. J Thromb Haemost. 2020;18(5):1094-9. https:// doi.org/10.1111/jth.14817.

16. Paranjpe I, Fuster V, Lala A, Russak AJ, Glicksberg BS, Levin MA, et al. Association of Treatment Dose Anticoagulation with in-Hospital Survival among Hospitalized Patients with COVID-19. J Am Coll Cardiol. 2020;76(1): 122-4. https://doi.org/10.1016/j.jacc.2020.05.001.

17. Cattaneo M, Bertinato EM, Birocchi S, Brizio C, Malavolta D, Manzoni M, et al. Pulmonary embolism or pulmonary thrombosis in COVID-19? Is the recommendation to use high-dose heparin for Thromboprophylaxis justified? Thromb Haemost. 2020;120(8):1230-2. https://doi.org/10.1055/s0040-1712097.

18. Pesavento R, Ceccato D, Pasquetto G, Monticelli J, Leone L, Frigo A, et al. The hazard of (sub) therapeutic doses of anticoagulants in non-critically ill patients with Covid-19: the Padua province experience. J Thromb Haemost. 2020;18(10):2629-35. https://doi.org/10.1111/jth.15022.

19. Yao Y, Cao J, Wang Q, Shi Q, Liu K, Luo Z, et al. D-dimer as a biomarker for disease severity and mortality in COVID-19 patients: a case control study. J Intensive Care. 2020;8(1):49. https://doi.org/10.1186/s40560-020-00466-z.

20. Charlson ME, Pompei P, Ales KL, MacKenzie CR. A new method of classifying prognostic comorbidity in longitudinal studies: development and validation. J Chronic Dis. 1987;40(5):373-83. https://doi.org/10.1016/0021-9681(87)90171-8.

21. Lambden S, Laterre PF, Levy MM, Francois B. The SOFA score-development, utility and challenges of accurate assessment in clinical trials. Crit Care. 2019; 23(1):374. https://doi.org/10.1186/s13054-019-2663-7.

22. Clinical management of severe acute respiratory infection (SARI) when COVID-19 disease is suspected: interim guidance. 2020. [https://www.who. int/publications-detail/clinical-management-of-severe-acute-respiratoryinfection-when-novel-coronavirus-(ncov)-infection-is-suspected]. Accessed 13 Mar 2020.

23. Pisters R, Lane DA, Nieuwlaat R, de Vos CB, Crijns HJ, Lip GY. A novel userfriendly score (HAS-BLED) to assess 1-year risk of major bleeding in patients with atrial fibrillation: the euro heart survey. Chest. 2010;138(5):1093-100. https://doi.org/10.1378/chest.10-0134.

24. Schulman S, Kearon C. Subcommittee on control of anticoagulation of the $\mathrm{S}$, standardization Committee of the International Society on T, Haemostasis. Definition of major bleeding in clinical investigations of antihemostatic medicinal products in non-surgical patients. J Thromb Haemost. 2005;3(4): 692-4. https://doi.org/10.1111/j.1538-7836.2005.01204.x.

25. Meng Y, Lu W, Guo E, Liu J, Yang B, Wu P, et al. Cancer history is an independent risk factor for mortality in hospitalized COVID-19 patients: a propensity score-matched analysis. J Hematol Oncol. 2020;13(1):75. https:// doi.org/10.1186/s13045-020-00907-0.

26. Lemos ACB, do Espirito Santo DA, Salvetti MC, Gilio RN, Agra LB, Pazin-Filho $A$, et al. Therapeutic versus prophylactic anticoagulation for severe COVID19: A randomized phase II clinical trial (HESACOVID). Thromb Res. 2020;196: 359-66.
27. Tremblay D, van Gerwen M, Alsen M, Thibaud S, Kessler A, Venugopal S, et al. Impact of anticoagulation prior to COVID-19 infection: a propensity score-matched cohort study. Blood. 2020;136(1):144-7.

28. Li J, Li Y, Yang B, Wang H, Li L. Low-molecular-weight heparin treatment for acute lung injury/acute respiratory distress syndrome: a meta-analysis of randomized controlled trials. Int J Clin Exp Med. 2018;11(2):414-22.

29. Poterucha TJ, Libby P, Goldhaber SZ. More than an anticoagulant: do heparins have direct anti-inflammatory effects? Thromb Haemost. 2017; 117(3):437-44. https://doi.org/10.1160/TH16-08-0620.

30. Mouhat B, Besutti M, Bouiller $\mathrm{K}$, et al. Elevated D-dimers and lack of anticoagulation predict PE in severe COVID-19 patients. Eur Respir J. 2020; 56(4):2001811. Published 2020 Oct 22. https://doi.org/10.1183/13993003.01 811-2020.

31. Al-Samkari H, Karp Leaf RS, Dzik WH, Carlson JCT, Fogerty AE, Waheed A, et al. COVID-19 and coagulation: bleeding and thrombotic manifestations of SARS-CoV-2 infection. Blood. 2020;136(4):489-500. https://doi.org/10.1182/ blood.2020006520.

32. Zhu W, He W, Guo L, Wang X, Hong K. The HAS-BLED score for predicting major bleeding risk in Anticoagulated patients with atrial fibrillation: a systematic review and meta-analysis. Clin Cardiol. 2015;38(9):555-61. https:// doi.org/10.1002/clc.22435.

33. Rief P, Raggam RB, Hafner F, Avian A, Hackl G, Cvirn G, et al. Calculation of HAS-BLED score is useful for early identification of venous thromboembolism patients at high risk for major bleeding events: a prospective outpatients cohort study. Semin Thromb Hemost. 2018;44(4): 348-52. https://doi.org/10.1055/s-0037-1607433.

34. Kooiman J, van Hagen N, Iglesias Del Sol A, Planken EV, Lip GY, van der Meer FJ, et al. The HAS-BLED score identifies patients with acute venous thromboembolism at high risk of major bleeding complications during the first six months of anticoagulant treatment. PLoS One. 2015;10(4):e0122520. https://doi.org/10.1371/journal.pone.0122520.

35. Desai AK, Pang J, Aparnath $M$, llowite J. Utility of inferior vena cava filters in severe pulmonary embolism, catheter-directed therapy in massive and submassive pulmonary embolism, and HAS-BLED score to determine risk of major hemorrhage in pulmonary embolism. Am J Respir Crit Care Med. 2016;193(11):1301-3. https://doi.org/10.1164/rccm.201508-1565RR.

36. Castini D, Persampieri S, Sabatelli L, Erba M, Ferrante G, Valli F, et al. Utility of the HAS-BLED score for risk stratification of patients with acute coronary syndrome. Heart Vessel. 2019;34(10):1621-30. https://doi.org/10.1007/s003 80-019-01405-1.

37. Hsieh MJ, Wang CC, Chen CC, Wang CL, Wu LS, Hsieh IC. HAS-BLED score predicts risk of in-hospital major bleeding in patients with acute non-ST segment elevation myocardial infarction. Thromb Res. 2015;136(4):775-80. https://doi.org/10.1016/j.thromres.2015.08.015.

38. Lip GY, Lin HJ, Hsu HC, Su TC, Chen MF, Lee YT, et al. Comparative assessment of the HAS-BLED score with other published bleeding risk scoring schemes, for intracranial haemorrhage risk in a non-atrial fibrillation population: the Chin-Shan community cohort study. Int J Cardiol. 2013; 168(3):1832-6. https://doi.org/10.1016/j.ijcard.2012.12.076.

39. Chan KH, Ka-Kit Leung G, Lau KK, Liu S, Lui WM, Lau CP, et al. Predictive value of the HAS-BLED score for the risk of recurrent intracranial hemorrhage after first spontaneous intracranial hemorrhage. World Neurosurg. 2014;82(1-2):e219-23. https://doi.org/10.1016/j.wneu.2013.02.070

40. Freixo C, Ferreira V, Goncalves J, Teixeira G, Antunes I, Veiga C, et al. HASBLED score predicts the risk of major bleeding in chronic anticoagulation after lower limb surgical revascularization. Ann Vasc Surg. 2019;59:190-4. https://doi.org/10.1016/.javsg.2019.01.022.

41. Konishi H, Miyauchi K, Tsuboi S, Ogita M, Naito R, Dohi T, et al. Impact of the HAS-BLED score on long-term outcomes after percutaneous coronary intervention. Am J Cardiol. 2015;116(4):527-31. https://doi.org/10.1016/j.a mjcard.2015.05.015.

\section{Publisher's Note}

Springer Nature remains neutral with regard to jurisdictional claims in published maps and institutional affiliations. 